\title{
Bonding Pathways of Gold Nanocrystals in Solution
}

Zainul Aabdin ${ }^{1,2,3,4}$, Jingyu Lu ${ }^{1,2,3,4}$, Utkarsh Anand ${ }^{1,2,4}$, N. Duane Loh ${ }^{1,3}$, Utkur Mirsaidov 1,2,3,4

1. Department of Physics, National University of Singapore, 2 Science Drive 3, Singapore, 117551.

2. Graphene Research Center, National University of Singapore, 6 Science Drive 2, Singapore, 117546.

3. Center for Bioimaging Sciences and Department of Biological Sciences, National University of Singapore, 14 Science Drive 4, Singapore, 117543.

4. NUSNNI-Nanocore, 5A Engineering Drive 1, National University of Singapore, Singapore 117411.

The bonding between nanocrystals in solution is one of the most important crystal growth pathways to the bottom-up assembly of nanocrystals into hierarchical structures for the fabrication of nanoscale devices and nanomaterials. The main challenge, however, is to understand and avoid defect formation at the bonding interfaces between nanocrystals. It is believed that any misalignment results in defect formation at bonding interface, and defect-free coherent bonding is only possible when bonding lattice planes are perfectly oriented. Recent studies employing in situ imaging showed that the dynamics of nanocrystal bonding in solution is rich. For the first time the pre-alignment of nanocrystals prior to attachment, post-bonding dipole-induced realignment of nanocrystals, and annealing of line dislocations in nanocrystals were observed [1-3]. However, two important key questions to nanocrystal bonding remain unresolved: 1) what are the necessary conditions for nanocrystal attachment in solution that will lead to defect-free coherent bonding? and 2) what is the likelihood of such coherent bonding? Here, we resolved the mechanisms underpinning these phenomena using liquid-cells developed for time-resolved electron microscopy [4]. Our study is the first to give a detailed description of two distinct bonding pathways which shows how attachment geometry of the system affects the bonding of nanocrystals with rotational and translational degrees of freedom [5].

Figure 1A shows the coherent bonding of two nanocrystals initially separated by $\sim 1 \mathrm{~nm}$, and their (111) lattice planes misaligned by $10^{\circ}$ (at $\mathrm{t}=6.9 \mathrm{~s}$ ) prior to contact. As the nanocrystals approach each other, their lattice misalignment decreases to $9^{\circ}$ upon contact (not shown here), then visibly realigns into coherent bonding (Fig. 1A: $\mathrm{t}=25.5 \mathrm{~s}$ and $48.2 \mathrm{~s}$ ). Here, the bonding was coherent because the common (111) lattice planes reflections of the nanocrystals merged into a single reflection with no visible defects (inset $\mathrm{t}=48.2 \mathrm{~s}$ ). Expectedly, defect-mediated bonding occurs when two nanocrystals meet with their (111) lattice planes initially misaligned by a large angle, for example by about $32^{\circ}$ in Figure $1 \mathrm{~B}$. Here the nanocrystals do not realign upon bonding and a visible defect forms at their merging interface $(\mathrm{t}=$ $6.9 \mathrm{~s}$, black arrow). Post bonding images and splitted Fourier reflections show that the newly formed nanoparticle is not a single crystal (Fig. 1B: $t=11.2 \mathrm{~s}$ ). The coherent and defect-mediated bonding sequences captured in Figure 1 are remarkable because upon contact the nanocrystals can reorient themselves to a limited extent, either to achieve coherent bonding when the two nanocrystals are initially slightly misaligned, or rotate to a mutual configuration that allows defects to form.

The experimental results in Figure $2 \mathrm{~A}$ show that there is a critical misalignment angle $\left(\beta_{\text {critical }} \sim 15^{\circ}\right)$ that separates two pathways for bonding: below this critical angle gold nanocrystals can realign for defectfree coherent bonding, and above which the defect are formed at the interface which mediate the bonding (Fig. 2B). Our results have significant implication for nanocrystal growth, and bottom-up design of hierarchical nanostructures [6]. 
[1] R. L. Penn and J.F. Banfield, Science, 281 (1998), p.969.

[2] Li. Dongsheng et al., Science, 336 (2012), p.1014.

[3] H. G. Liao et al., Science, 336 (2012), p.1011.

[4] U. M. Mirsaidov et al., P. Proc. Natl. Acad. Sci. U.S.A., 109 (2012), p.7187.

[5] Z. Aabdin et al., Nano Letters, 14 (2014), p.6639.

[6] This work was supported by the Singapore National Research Foundation's Competitive research program funding (NRFCRP9-2011-04). The authors acknowledge the support of the Electron Microscopy Facility at Center for Bioimaging Science, National University of Singapore.
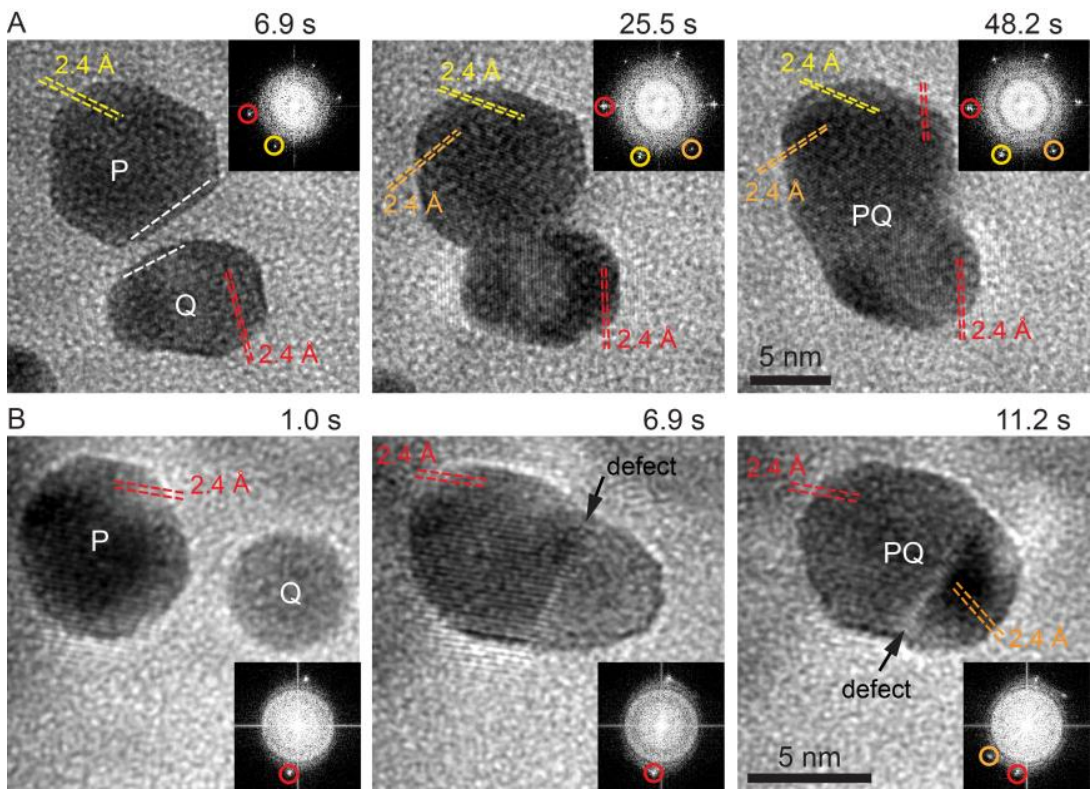

Figure 1. Time resolved images of (A) coherent and (B) non-coherent bonding between two gold nanocrystals (P and Q). Coherent bonding shares a common (111) lattice planes that are misaligned by only $\sim 9^{\circ}$ at contact yields defect free nanocrystal PQ, whereas, for non-coherent bonding (111) lattice planes are misaligned by $32^{\circ}$ at contact yields a nanocrystal PQ with defect at the bonding interface. Lattices fringes along (111) plane with $2.4 \AA$ spacing are marked by dashed parallel lines with corresponding reflections circled in the Fourier transform of the image as shown in the inset
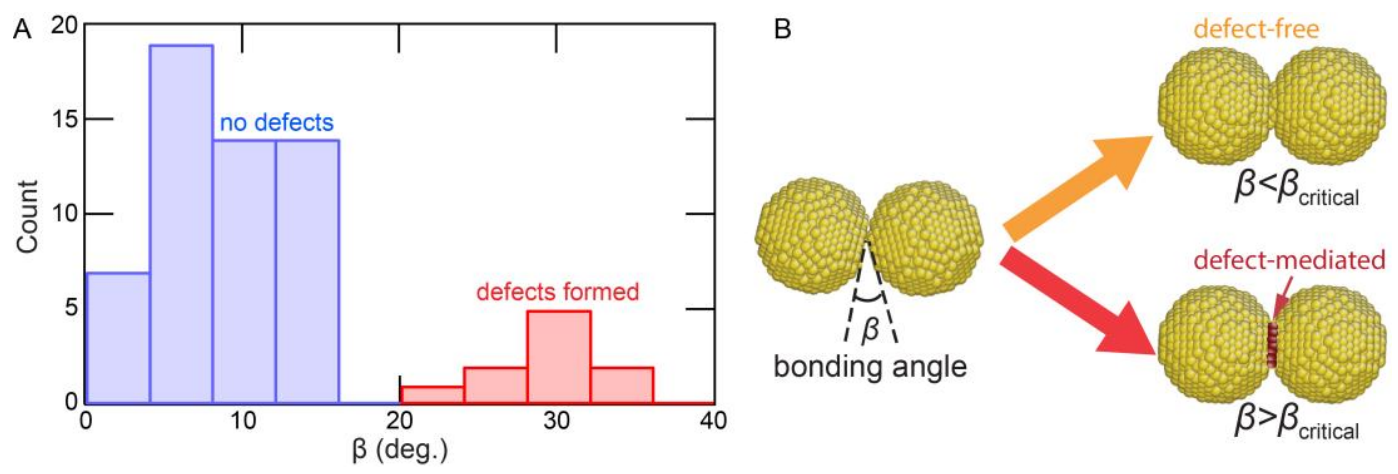

Figure 2. (A) Histogram of experimental nanocrystal bonding events at different orientation angles ( $\beta$ ) measured between the common (111) lattice planes at contact as shown in schematic diagram (B). At $\beta$ $\leq 15^{\circ}$ ( $\beta_{\text {critical }}$ ), the bonding between two gold nanocrystals results in defect-free single nanocrystals, whereas, for angles between $20^{\circ}$ and $35^{\circ}$ clear persistent defects at the merging interface are observed. 